\title{
Importância da Correlação Clínico-Radiográfica na Aproximação Diagnóstica das Cardiopatias Congênitas
}

\author{
Edmar Atik
}

São Paulo, SP

Em face dos indiscutíveis avanços diagnósticos das cardiopatias congênitas e adqüiridas, que compõem a cardiologia pediátrica ${ }^{1-3}$, pergunta-se da importância, hoje, da radiografia de tórax, como um método que outrora preponderava, conjuntamente, com o eletrocardiograma e os elementos clínicos, para a obtenção do diagnóstico funcional dessas cardiopatias.

De fato, as etapas diagnósticas foram se sucedendo através os tempos. Conta a história, que os elementos clínicos, inicialmente, eram correlacionados aos da anatomia patológica e, percebendose então, retrospectivamente, por exemplo, a característica do sopro peculiar da mínima comunicação interventricular, conhecido hoje como o sopro de Roger ${ }^{4}$. Posteriormente, esses elementos foram se correlacionando com os da eletrocardiografia e radiografia de tórax em um tripé semiológico cujos diagnósticos anatômico e funcional eram ultimados sempre pelo estudo hemodinâmico.

Com o advento do ecocardiograma e de suas imagens diagnósticas indiscutíveis, estas vieram a substituir o estudo hemodinâmico nesta definição anatômica e funcional, alterando, assim, os marcos diagnósticos e, conseqüentemente, a importância da semiologia e a conduta adotadas na especialidade.

Estas imagens, hoje, são tão aceitas que servem até de guias e de testes finais, mesmo após a correção de defeitos, ainda na sala operatória, através o método transesofágico.

Pergunta-se, portanto, novamente, se o método radiográfico em sua correlação clínica foi ultrapassado e se tornou realmente obsoleto?

Se partirmos da premissa de que nenhum método é considerado inteiramente soberano na determinação de todas as variáveis anatômicas e funcionais, ainda a radiografia de tórax em conjunto com a clínica serve de guia funcional a um entendimento fisiopatológico inicial, antes de se poder estabelecer, de maneira mais definida, o diagnóstico.

Assim, seria este método um guia para: 1) obter-se um entendimento preliminar do tipo da cardiopatia em questão; 2) encetar o raciocínio clínico do tipo da anomalia em estudo, se com balanceamento de fluxos sistêmico-pulmonar ou não; 3) estabelecer um teste diagnóstico para se poder argumentar em possíveis diagnósticos equivocados, através a ecocardiografia; 4) por fazer parte do diagnóstico clínico, aliado aos elementos clínico-semiológicos

Instituto do Coração do Hospital das Clínicas da Faculdade de Medicina da Universidade de São Paulo

Endereço para correspondência: Edmar Atik - Instituto do Coração da FMUSP Av. Enéas Carvalho de Aguiar 44 - Cep 05403-000 - São Paulo - SP E-mail: conatik@incor.usp.br

Enviado para publicação em 27/05/2004

Aceito em 22/06/2004 e eletrocardiográficos, é tido como elemento fundamental e, assim, também indispensável; 5) estimular o raciocínio clínico, este sim primordial para se obter o entendimento da dinâmica da cardiopatia em questão e, portanto, da conduta a ser tomada.

Com essas premissas torna-se de risco não fundamentar a conduta sob os elementos clínicos, pois ao clínico se reserva a conduta abalizada e pensada, ainda mais que a cardiologia pediátrica exige raciocínio na estimativa e aproximação do diagnóstico e, principalmente, na conduta. Torna-se um erro, aliás grosseiro, a análise isolada dos elementos ecográficos para deles orientar a indicação cirúrgica, a qual realizada diretamente com base nestes elementos ou, ainda, pelo pensamento do próprio grupo cirúrgico, pode resultar em enganos ou em eventos precipitados e desnecessários.

O clínico avalia os elementos clínico-semiológicos, em conjunto com os do eletrocardiograma e da radiografia de tórax, os contrabalança aos do risco cirúrgico e aos da presuntiva evolução pósoperatória subseqüente, para se aquilatar na ocasião a época ideal da indicação operatória de cada anomalia, analisada dentro de um universo de possibilidades, tão peculiar à especialidade.

Em contraposição são assistidos, hoje, descalabros e esperanças ilusórias que devem ser certamente eliminados, como por exemplo, indicações operatórias de comunicação interatrial sem repercussão, ainda no $1^{\circ}$ ano de vida, correção de comunicação interventricular discreta nos dois primeiros anos com possibilidade ainda do fechamento espontâneo, dentre outras situações.

Essa maior liberação para a operação, realizada por profissionais não vinculados à "preocupação clínica", está sendo respaldada certamente nos progressos obtidos, como os relacionados a riscos cirúrgicos cada vez menores e a diagnósticos mais corretos através as imagens ecocardiográficas.

De fato, esses avanços são conquistas que verdadeiramente devem ser interpostas em benefício real do paciente, sem no entanto, não ultrapassar os passos iniciais de análise clínica cuidadosa.

Por isso deve-se resgatar novamente os elementos essenciais e fundamentais através a continuidade da correlação clínica.

Ademais, cito alguns outros exemplos da utilidade da radiografia de tórax, na prática clínica, que reforçam a necessidade de que a correlação clínica deva estar sempre presente nas discussões diagnósticas e de condutas. Pode-se afirmar que essa falta de correlação resulte em equívocos, que na literatura não são mencionados com ênfase, talvez por que devam ser omitidos prioritariamente pelo próprio avanço indiscutível da ecocardiografia que sobrepuja as limitações conhecidas do método radiográfico.

No entanto, lembro que através uma radiografia simples de tórax podemos facilmente descartar o diagnóstico da comunicação interatrial que, equivocadamente, é realizado por vezes pela eco- 
cardiografia e que, muitas vezes, orienta daí para indicações operatórias descabidas. Ademais, aquilata funcionalmente a repercussão de comunicações interventriculares, que por vezes podem ser avaliadas pelo ecocardiograma como de grandes dimensões, mas que, funcionalmente, são pequenas em face de obstrução parcial pelo tecido tricúspide interposto. A radiografia de tórax também avalia, funcionalmente, o grau de obstrução ao fluxo pulmonar em cardiopatias isoladas, como na estenose pulmonar ou ainda em associação a outros defeitos, como no ventrículo único, por exemplo. Nessas condições, o encontro de gradientes variados de um para outro exame ecocardiográfico nos permite privilegiar os dados clínicos para a determinação da conduta posterior. 0 mesmo pensamento pode ser dirigido para casos com obstrução do lado esquerdo do coração, como na estenose aórtica e na coartação da aorta. Não querendo ser presunçoso mas, pode ele até questionar dados de função cardíaca mal interpretados pela ecocardiografia ou ainda por outros métodos, quando se diagnostica disfunção miocárdica em contraposição nítida radiográfica, mostrando área cardíaca de dimensões próximas do normal.

Acrescendo o fato de ser a radiografia de tórax exame de baixo custo e consolidando-o dentro do fundamental orienta até para a necessidade ou não da execução em determinados casos da própria ecocardiografia, esta tida no entanto como indispensável hoje no diagnóstico anatomofuncional das cardiopatias na infância.

Como a radiografia de tórax pode ser considerada como método realmente útil? Vale análise para a consideração a respeito do que este exame pode oferecer concretamente. Neste contexto, a radiografia de tórax nos mostra, como todo método, aspectos anatômicos e funcionais ${ }^{5-8}$. Os funcionais são obtidos a partir da análise adequada do tipo da vascularidade pulmonar, que pode se apresentar com características normais, diminuida em calibre e aumentada, por hiperfluxo pulmonar ou por congestão venocapilar pulmonar.

Desta maneira, a trama vascular pulmonar intra-parenquimatosa reflete muito a dinâmica cardiovascular que acompanha os vários tipos de cardiopatias congênitas, tornando-se o alvo da interpretação inicial em uma radiografia de tórax, antes mesmo da análise da área cardíaca.

Assim, quando a trama vascular pulmonar é considerada normal em calibre e em distribuição denota que os fluxos sistêmico e pulmonar estão mais ou menos balanceados, o que ocorre nas cardiopatias congênitas obstrutivas direitas e esquerdas que, ainda por mecanismos adequados de compensação, mantêm o débito anterógrado de sangue. Ainda mais, pode ocorrer também em cardiopatias com descompensação cardíaca, que ainda mantém um débito cardíaco adequado, como nas miocardiopatias de qualquer natureza etiológica.

A vascularidade pulmonar normal se distribui desde os hilos até $2 / 3$ internos dos campos pulmonares, não sendo visível no restante $1 / 3$ externo, com dominância das imagens para os lobos inferiores em relação aos superiores. 0 calibre vai se adelgaçando desde o hilo, obedecendo as bifurcações dos vários segmentos pulmonares à direita e à esquerda, sendo em número de 10 de cada lado, três para os lobos superiores, dois para os lobos médio direito e da língula à esquerda e cinco para os lobos inferiores.

Essa trama vascular se torna de dimensões diminuidas com esta mesma distribuição descrita, em face da presença de hipofluxo pulmonar, que acompanha o primeiro grupo das cardiopatias cianogênicas com obstrução ao fluxo pulmonar e/ou regurgitação de sangue em nível valvar, tricúspide ou pulmonar. Este tipo de trama vascular se apresenta também nas cardiopatias acianogênicas com obstrução à direita do coração, quando o fluxo anterógrado não é mais mantido.

Por outro lado, quando há hiperfluxo pulmonar, nota-se aumento do diâmetro dos vasos, normalmente, distribuídos pelos dois pulmões e ocorre nas cardiopatias acianogênicas com desvio de sangue da esquerda para a direita e nas cardiopatias cianogênicas sem estenose pulmonar.

$\mathrm{O}$ aspecto congestivo pulmonar com aumento da trama vascular pulmonar, decorrente de engurgitamento hilar e dos lobos superiores, observa-se em cardiopatias que ocasionem hipertensão venocapilar pulmonar, como ocorre em lesões obstrutivas à esquerda ou em qualquer situação anatomofuncional que ocasione disfunção do ventrículo esquerdo.

Na seqüência desta análise radiográfica associa-se a existência ou não de cardiomegalia a cada tipo de vascularidade pulmonar, acrescentando, assim, o aspecto anatômico do defeito para se alcançar o diagnóstico definitivo.

Assim, com trama vascular pulmonar normal, as cardiopatias congênitas, que se acompanham de área cardíaca também normal, são representadas por defeitos acianogênicos com muito discretos desvios de sangue da esquerda para a direita (comunicações interatrial e interventricular e canal arterial), lesões obstrutivas discretas, que ainda preservam um fluxo anterógrado normal (estenoses pulmonar e aórtica e coarctação da aorta), anomalias das artérias coronárias, como aneurisma e/ou estenose congênitas ou adqüiridas, síndrome de Eisenmenger, anomalias do arco aórtico e outras cardiopatias que preservam fluxos pulmonar e sistêmico balanceados.

Com trama vascular pulmonar normal, mas com cardiomegalia, são lembradas as lesões obstrutivas à esquerda do coração que não mantêm fluxo anterógrado normal, mas sem descompensação acentuada, tumores cardíacos, insuficiência valvar mitral sem congestão venocapilar pulmonar, hipertensão pulmonar com insuficiência tricúspide.

Com trama vascular pulmonar diminuída e com área cardíaca normal, citam-se as cardiopatias obstrutivas à direita e que não mantêm o fluxo anterógrado normal. Dentre as acianogênicas (estenose valvar, supravalvar e subvalvar pulmonar, estenose de via de entrada de ventrículo direito) e dentre as cianogênicas com obstrução ao fluxo pulmonar (tetralogia de Fallot, tipos de atresia tricúspide, atresia pulmonar com septo ventricular íntegro e com comunicação interventricular, ventrículo único, dupla via de saída de ventrículo direito e de ventrículo esquerdo, transposição corrigida das grandes artérias, transposição das grandes artérias).

Com trama vascular pulmonar diminuida e com cardiomegalia, destacam-se as cardiopatias obstrutivas à direita, acompanhadas de insuficiência valvar tricúspide ou da valva atrioventricular única. Neste contexto figuram, dentre as acianogênicas, a estenose pulmonar valvar e o defeito do septo atrioventricular e nas cardiopatias cianogênicas, tipos de atresia pulmonar com septo ventricular íntegro, tetralogia de Fallot e dupla via de saída de ventrículo direito com defeito do septo atrioventricular, tipos de atresia tricúspide com septo interatrial restritivo ou com insuficiência mitral e insuficiência ventricular esquerda. A ocorrência de insuficiência tricúspide e desvio de sangue da direita para a esquerda em nível atrial, como ocorre na anomalia de Ebstein, constitui outro me- 
canismo patogênico deste grupo, explicando a diminuição da vascularidade pulmonar e a cardiomegalia.

Com trama vascular pulmonar aumentada por congestão pulmonar e área cardíaca normal, destacam-se as cardiopatias obstrutivas do lado esquerdo do coração como a estenose mitral, cor triatriatum, estenose de veias pulmonares, ausência de conexão atrioventricular esquerda, drenagem anômala total das veias pulmonares com obstrução ao fluxo venoso pulmonar, hipoplasia do coração esquerdo com comunicação interatrial restritiva e associações de defeitos com obstruções em nível da valva mitral, como na dupla via de saída de ventrículo direito, ventrículo único e síndrome de Shone.

Com trama vascular pulmonar aumentada por congestão pulmonar e cardiomegalia, salientam-se as cardiopatias acianogênicas que levam à insuficiência ventricular esquerda por sobrecarga de volume, como na insuficiência valvar mitral, insuficiência valvar aórtica, e insuficiência da valva atrioventricular esquerda na transposição corrigida das grandes artérias, ou por sobrecarga de pressão em lesões obstrutivas, como na estenose aórtica e coarctação da aorta. Mencionam-se ademais as miocardiopatias dilatadas de ventrículo esquerdo de origem isquêmica (origem anômala da artéria coronária esquerda) ou de origem arrítmica ou mesmo infecciosa.

Com trama vascular pulmonar aumentada por hiperfluxo e com área cardíaca normal, figuram as cardiopatias que mantêm um fluxo pulmonar discretamente aumentado sem provocarem sobrecarga de volume que aumente a área cardíaca. Neste grupo se inserem cardiopatias em geral cianogênicas com associação à estenose pulmonar, como a transposição completa das grandes artérias, tetralogia de Fallot, ventrículo único, dupla via de saída de ventrículo direito, agenesia da valva pulmonar. Ademais, cardiopatias que se acompanham de hipertensão pulmonar, como na transposição das grandes artérias associada à comunicação interventricular, a atresia aórtica com comunicação interventricular, a hipoplasia arterial pulmonar unilateral e a própria doença vascular pulmonar. Outra anomalia representativa deste tipo radiográfico é a transposição das grandes artérias com comunicação interatrial.

Com trama vascular pulmonar aumentada por hiperfluxo e cardiomegalia, destacam-se as cardiopatias com desvio de sangue da esquerda para a direita e conseqüente sobrecarga de volume e dentre as acianogênicas, a comunicação interatrial, a comunicação interventricular, o defeito do septo atrioventricular total e parcial, o canal arterial, a janela aortopulmonar, as fístulas arteriovenosas, a transposição corrigida das grandes artérias associada à comunicação interventricular. Das cianogênicas, a transposição das grandes artérias, o tronco arterial comum, a drenagem anômala total das veias pulmonares, hipoplasia do coração esquerdo com comunicação interatrial não restritiva, ventrículo único, atresia das valvas atrioventriculares direita e esquerda sem estenose pulmonar, dupla via de saída de ventrículo direito, atresia pulmonar com comunicação interventricular, cardiopatias complexas tipo criss-cross e isomerismos atriais.

Estes achados e encaminhamentos diagnósticos através a radiografia de tórax, aliados aos do eletrocardiograma e, principalmente aos elementos clínico-semiológicos, constituem-se o "essencial clínico" fundamentando a impressão inicial da cardiopatia e consubstanciando a idéia fisiopatológica e patogênica desses defeitos e que certamente vão ultimar a conduta a ser posteriormente estabelecida.

Esse diagnóstico clínico, hoje, sofre a confirmação obrigatória do estudo ecocardiográfico, o qual tem substituido nesta função e com vantagens o cateterismo cardíaco que, cada vez mais, tem sido orientado ao campo da intervenção terapêutica, substituindo a cirurgia cardíaca em muitas anomalias.

Desta maneira, segundo estimativas a cirurgia cardíaca corretiva tem sido realizada em aproximadamente $85 \%$ dos casos sem estudo hemodinâmico prévio, baseados na imagem ecocardiográfica ${ }^{9,10}$. A ressonância nuclear magnética tem auxiliado ultimamente essa tendência ainda mais que sua aplicação maior reside exatamente nas limitações da ecocardiografia ${ }^{11}$.

No entanto, reenfatizo que apesar dos avanços diagnósticos, é mera suposição de que os elementos clínicos tenham perdido importância. Através deles realiza-se o diagnóstico funcional, capital para se estabelecer a conduta, aprimoram-se as outras técnicas pela indagação e correlação dos achados e interage-se com todos os elementos diagnósticos, afim de se determinar dessas funções a conduta mais adequada.

Por tudo, pode-se afirmar que na conduta geral das cardiopatias congênitas, os elementos clínicos são necessários e fundamentais e os exames com imagens, indiscutíveis.

\section{Referências}

1. Bengel FM, Schwaiger M. Nuclear medicine studies of the heart. Eur Radiol. 1998; 8: 1698-706.

2. Davis CP, McKinnon GC, Debatin JF, von Schulthess GK. Ultra-high-speed MR imaging. Eur Radiol. 1996;6: 297-311.

3. Randolph GR, Hagler DJ, Khandheria BK, et al. Remote telemedical interpretation of neonatal echocardiograms: impact on clinical management in a primary care setting. J Am Coll Cardiol. 1999; 34: 241-5.

4. Roger $\mathrm{H}$. Recherches cliniques sur la communication congenitale des deux coeurs, par innoclusion de septum interventriculaire. Bulletin de l'Academie de Medecin. 1879;8:1077-85.

5. Barón H, CarrilloG, Diaz G: Radiologia. En Diaz G, Sandoval N, Vélez JF, Carrillo G: Cardiologia Pediátrica, $1^{\text {a }}$ edición, Editora McGraw Hill, Colombia, 2003; 77-89.
6. Medeiros So JH: Radiologia do Coração. Sarvier Editora, São Paulo, 1980; 513

7. Ovitt TW. The Chest Roentgenogram. In Emmanouilides GC, Allen HD, Riemenschneider TA, Gutgesell HP-Moss and Adams Heart Disease in Infants, Children and Adolescents. Williams \& Wilkins, fifth edition, Baltimore,1998; 182-190.

8. Soto B. Radiografia simple en las cardiopatías congénitas. En Sánchez PA: Cardiologia Pediátrica. Clínica Y Cirugia. Salvat Editores AS, Barcelona, 1986; 111-20.

9. Buendia Hernandez A, Gloss G. Management of congenital cardiopathies: state of the art. Arch Cardiol Mex. 2003;73:21-5.

10. Gidding SS, Anisman P. What pediatric residents should learn about congenital heart disease. Pediatr Cardiol. 2003;24:418-23.

11. Pignatelli RH, McMahon CJ, Chung T, Vick GW $3^{\text {rd }}$. Role of echocardiography versus MRI for the diagnosis of congenital heart disease. Curr Opin Cardiol. 2003;18:357-65. 